\title{
Deterministic Structures in Fractionated Atrial Electrograms During Human Persistent Atrial Fibrillation
}

\author{
Tiago P Almeida ${ }^{1,2}$, Fernando S Schlindwein ${ }^{3}$, João L Salinet ${ }^{2}$, Xin Li ${ }^{3}$, Gavin S Chu ${ }^{3}$, \\ Jiun H Tuan ${ }^{3}$, Peter J Stafford ${ }^{3}$, G André $\mathrm{Ng}^{3}$, Diogo C Soriano ${ }^{2}$ \\ ${ }^{1}$ Instituto Tecnológico de Aeronáutica, São José dos Campos, Brazil \\ ${ }^{2}$ Federal ABC University, São Bernardo do Campo, Brazil \\ ${ }^{3}$ University of Leicester, Leicester, United Kingdom
}

\begin{abstract}
In the present work, we sought to uncover possible deterministic dynamics in atrial tissue activations during persistent atrial fibrillation (persAF) using recurrence plots $(R P)$ and recurrence quantification analysis (RQA). 797 bipolar atrial electrograms (AEGs) were collected from 18 persAF patients undergoing pulmonary vein isolation (PVI). Automated AEG classification was performed using the CARTO criteria (Biosense Webster). Four RQA attributes were obtained from the AEGs: determinism (DET); the recurrence rate (RR); the laminarity (LAM); and the Shannon entropy of the diagonal line in a RP (ENTR). PVI significantly increased the four RQA features ([Mean $\pm S D$ ], DET: $87.7 \pm 8.3 \%$ vs. $90.7 \pm 8.5 \%$; RR: $60.2 \pm 17.3 \%$ vs. $63.6 \pm 17.4 \%$; LAM: $93.9 \pm 4.7 \%$ vs. 95.3 $\pm 5.5 \%$; ENTR: $3.9 \pm 0.6$ vs. $4.2 \pm 0.8, P \leq 0.0001)$. The attributes were higher for normal AEGs (normal vs. fractionated, DET: $90.5 \pm 8.7 \%$ vs. $86.7 \pm 7.6 \%$; RR: $64.0 \pm 18.0 \%$

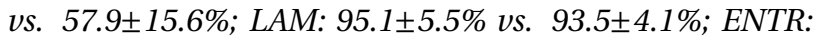
$4.2 \pm 0.8$ vs. $3.8 \pm 0.5, P \leq 0.0001$ ). Our results suggest that the increase of irregular activations on fractionated AEGs in persAF was not associated to a random structure. Diagonal structures associated to deterministic behaviour were still present in RPs from fractionated AEGs, leading to a high residual determinism, which could be related to unstable periodic orbits in a chaotic motion.
\end{abstract}

\section{Introduction}

Atrial fibrillation (AF) is the most common sustained arrhythmia found in the clinical practice [1]. Pulmonary vein (PV) isolation (PVI) through radiofrequency catheter ablation is regarded as the cornerstone for AF ablative therapy, but it has been shown insufficient for persistent $\mathrm{AF}$ (persAF) treatment due to extensive atrial remodelling. Different methods have been introduced to identify such remodelled atrial substrates to guide persAF ablation - such as fractionated atrial electrograms (AEGs) and functional re-entries with promising initial results [2,3]. More recent works, however, could not reproduce such results $[4,5]$, possibly due to the existence of multiple mechanisms participating - either simultaneously or intermittently - in its perpetuation [2,3,6], methodological heterogeneities [7], and poor understanding of the underlying AF dynamics $[4,6,8,9]$. Therefore, biological markers that better explain $\mathrm{AF}$ behaviour to guide ablation are still in debate.

\subsection{Recurrence analysis}

Recurrence plots (RPs) consist of a two-dimensional graphical technique used for the study of the topological structure of solutions of multidimensional dynamic systems [10]. It helps to evaluate the reappearance of states in a multidimensional vector from the construction of a binary distance matrix, associating the value 1 whenever one point in the multidimensional vector is close to another point by a distance less than $\varepsilon$, and the value 0 otherwise. This binary matrix can be represented graphically by associating a black pixel to the value 1 . The RP relates to the regularity and stationarity characteristics of the signal/system, as well as to its dynamic properties, such as the determinism of the state vector. Recurrence quantification analysis (RQA) establishes statistical attributes considering the distribution of diagonal and vertical lines of different sizes in the RP in order to characterize the structure and regularity of the observation [11]. It has been systematically used in biomedical signals processing and systems analysis in general $[12,13]$.

In the present study, we investigated the presence of possible deterministic dynamics in AEGs collected during persAF using RP and RQA. 


\section{Methods}

\subsection{Electrophysiological Study}

The population consisted of 18 patients (16 male; mean age $56.1 \pm 9.3$ years; history of AF $67.2 \pm 45.6$ months) referred to Glenfield Hospital, UK, for first time catheter ablation of persAF. Details of the clinical characteristics of the study subjects and the mapping procedure have been provided elsewhere [14]. All patients were in $\mathrm{AF}$ at the start of the procedure. Study approval was obtained from the local ethics committee and all procedures were performed with full informed consent.

All antiarrhythmic drugs, except amiodarone, were discontinued for at least 5 half-lives before the start of the procedure. 3D LA geometry was created within Ensite $\mathrm{NavX}^{\mathrm{TM}}$ (St. Jude Medical, St. Paul, Minnesota) using a deflectable, variable loop circular PV mapping catheter (Inquiry Optima, St. Jude Medical). PVI was performed with a point-by-point wide area circumferential ablation approach, followed by the creation of a single roof line (Cool Path Duo irrigated RF catheter, St. Jude Medical). No additional AEG-guided ablation was performed in this study. Sequential point-by-point bipolar AEGs were collected from 15 pre-determined atrial regions before and after LA ablation. All patients were in AF before and after ablation during signal collection.

A total of 797 AEGs were recorded from the LA, 455 before and 342 after PVI, with a sampling frequency of 1200 $\mathrm{Hz}$, and band-pass filtered within 30-300 Hz. Each AEG was exported from NavX with $8 \mathrm{~s}$. AEG classification was performed following the CARTO criteria (Biosense Webster, Diamond Bar, California): ICL $\geq 4, \mathrm{ACI} \leq 82 \mathrm{~ms}$ and $\mathrm{SCI} \leq 58 \mathrm{~ms}$ [7].

\subsection{Recurrence quantification analysis}

The state space map was reconstructed for each AEG following the Taken's reconstruction [10], which permits inferring on possible deterministic structures from unidimensional observations through the construction of auxiliary axes. These axes were estimated considering delayed samples of the unidimensional observation $\mathbf{x}(k)$, such as:

$$
\mathbf{x}(k)=\left[x(k), x(k-\tau), \ldots, x\left(k-\left(d_{e}-1\right) \tau\right)\right]
$$

where $d_{e}$ is the embedding dimension (number of coordinates used for the $\mathbf{x}(k)$ representation, and $\tau$ represents the time-delay embedding among samples (Figure $1)$.

The RP coordinate $\mathrm{R}_{i, j}$ is defined based on the distance between the respective states $\mathbf{x}_{i}$ and $\mathbf{x}_{j}$ considering an arbitrary threshold $\varepsilon$ following the reconstruction,
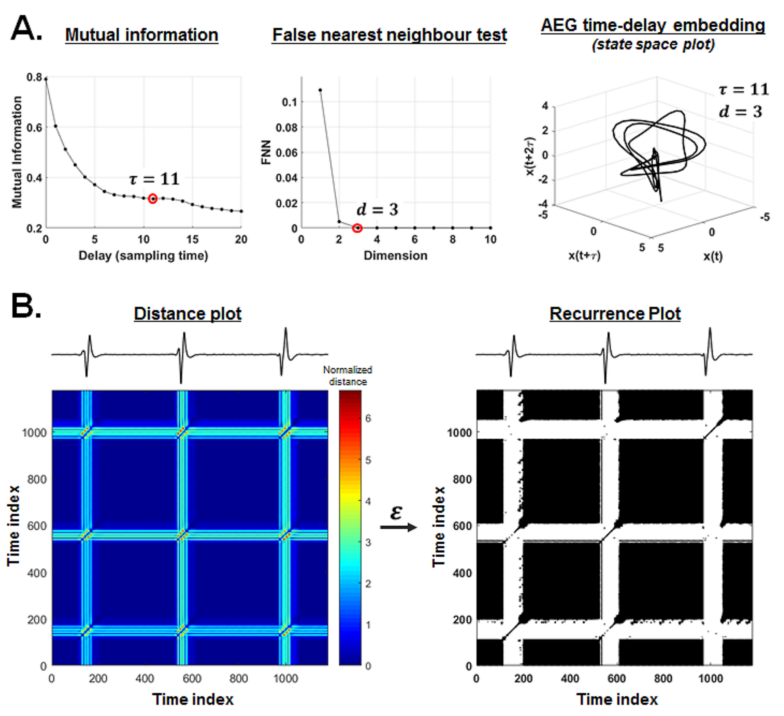

Figure 1: A. AEG state space reconstruction following the Takens' theorem, time-delay embedding and embedding dimension; B. Distance plot based on state space map (left), and the resulting RP after an adaptive threshold $\varepsilon$ was applied on the distance plot (right). The AEG is shown above both the distance plot and the RP.

which can be expressed as:

$$
R_{i, j}(\varepsilon)=\Theta\left\{\varepsilon-\left\|\mathbf{x}_{i}-\mathbf{x}_{j}\right\|\right\}
$$

where $\Theta\{\cdot\}$ refers to the Heaviside function.

The binary pattern observed in a RP is intrinsically related to the generative dynamics of the observation: the horizontal structures are associated with the laminarity (or turbulence) of the signal; the diagonal structures reflect the co-evolution of states, and indicate possible deterministic dynamics. The RQA is associated with statistical measures representative of the matrix $R_{i, j}$, as detailed by Marwan and colleagues [10]. In the present study we used:

- The recurrence rate (RR), which represents the density of points in a RP:

$$
R R=\frac{1}{N^{2}} \sum_{i, j=1}^{N} R_{\mathrm{i}, \mathrm{j}}
$$

- The ratio between recurrence points that form diagonal structures (with length $l \geq l_{\text {min }}$ ) is referred to as determinism (DET):

$$
D E T=\frac{\sum_{l=l_{\min }}^{N} l P(l)}{\sum_{l=1}^{N} l P(l)}
$$

- The ratio between recurrence points that form vertical structures (with length $v \geq v_{\text {min }}$ ) is referred to as laminarity (LAM):

$$
L A M=\frac{\sum_{v=v_{\min }}^{N} v P(v)}{\sum_{v=1}^{N} v P(\nu)}
$$


- Shannon entropy of diagonal lines in a RP (ENTR) reflects the RP's complexity regarding the diagonal lines:

$$
E N T R=\sum_{l=l_{\min }}^{N} P(l) \ln P(l)
$$

\subsection{Statistics}

All values are expressed as median \pm interquartile range. Non-parametric unpaired data were analysed using the Mann-Whitney test. Receiver operating characteristic (ROC) curves were created using the CARTO criteria as the reference for AEG classification and the RQA attributes as discriminators. The optimum sensitivity and specificity on the ROC curve was defined as the point on the curve with the shortest distance to the top left corner of the graph. Validation of the optimum point of operation for AEG classification was performed with leave-one-out cross-validation. P-values of less than 0.05 were considered statistically significant.

\section{Results}

Figure 2 illustrates two typical AEGs, one normal and one fractionated, and their respective RPs. The RPs from normal AEGs show big blocks of recurrence, suggesting high laminarity and determinism. Although those recurrence blocks were deteriorated, the RPs from fractionated AEGs revealed remaining diagonal lines, suggesting the presence of residual determinism.
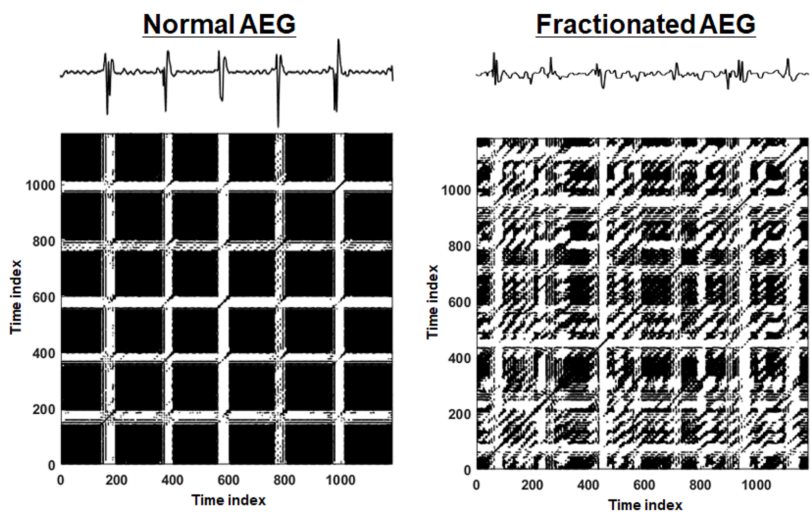

Figure 2: Two AEGs and their respective RPs: a normal AEG (left) and a fractionated AEG (right).

It is known that PVI affects electrophysiologic properties of the atrial tissue, even in regions distant to the ablation sites [15]. These changes were captured by the four RQA attributes, as illustrated in Figure 3, which suggest these features are capturing relevant electrophysiologic information. The four attributes significantly increased after ablation, suggesting that the AEGs became more organized following PVI.
As expected given their periodic and regular behaviour, the RQA-based features were significantly higher for normal AEGs when compared to fractionated AEGs (Figure 4). However, fractionated AEGs demonstrated a high residual determinism, which suggest they were not associated to a random structure.
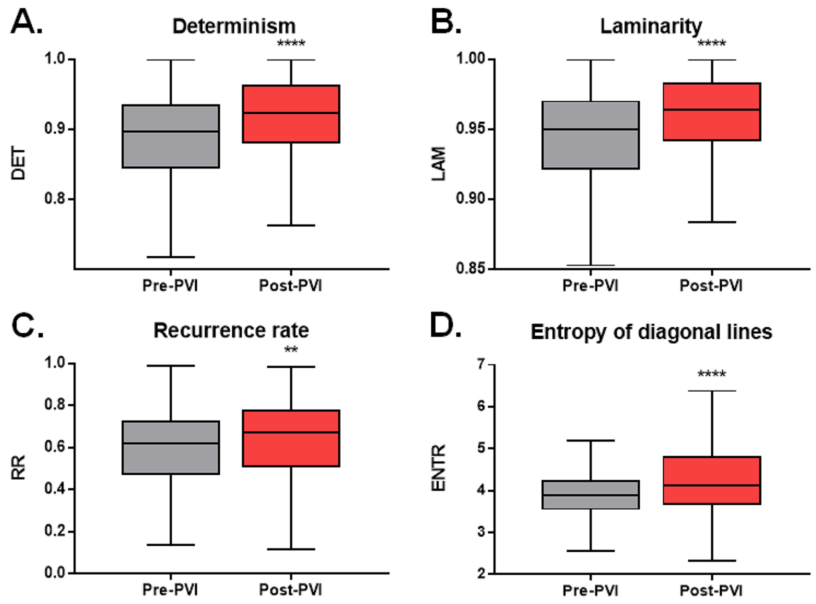

Figure 3: The RQA-based features before and after PVI. A. DET, B. RR, C. LAM, D. ENTR. ${ }^{* *} \mathrm{P} \leq 0.01,{ }^{* * *} \mathrm{P} \leq 0.0001$
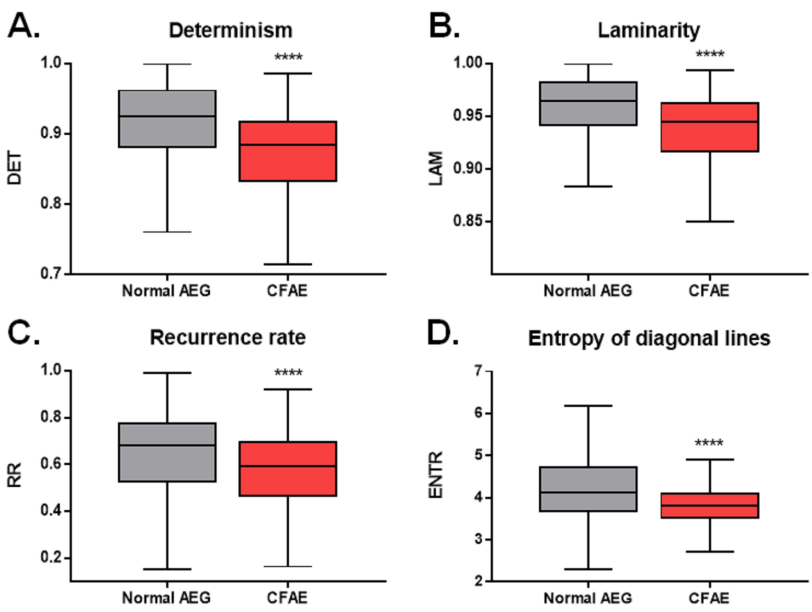

Figure 4: The RQA-based features for normal and fractionated AEGs. A. DET, B. RR, C. LAM, D. ENTR. **** $\mathrm{P} \leq 0.0001$

The ROC curves (Table 1) suggest that the RQA-based attributes can be used to effectively discriminate normal AEGs from fractionated ones.

\section{Discussion and conclusion}

The present work represents an initial step towards the characterization of AEGs during persAF using RQAbased features. The results suggest that the increase of 
Table 1: ROC curves for the AEG discrimination using RQA-based features.

\begin{tabular}{lccccc}
\hline \hline RQA-Feature & Thr & Sens & Spec & AUC & Val (\%) \\
\hline DET & 0.919 & 0.77 & 0.44 & 0.70 & 64 \\
RR & 0.663 & 0.68 & 0.46 & 0.62 & 59 \\
LAM & 0.959 & 0.71 & 0.41 & 0.69 & 63 \\
ENTR & 4.025 & 0.72 & 0.45 & 0.66 & 61 \\
\hline \hline
\end{tabular}

Abbreviations: Thr - optimum point of operation; Sens - sensitivity; Spec - specificity; AUC - area under the curve; Val - leave-one-out cross-validation. $\mathrm{P} \leq 0.0001$ for all curves.

irregular activations on fractionated AEGs was not necessarily associated to a random structure. On the contrary, RQA has revealed a residual amount of determinism, which could be related to unstable periodic orbits in repeating patterns of activations, indicating the presence of mechanisms recurring intermittently in preferred atrial regions during persAF, in line with recent findings $[6,9]$.

\section{Acknowledgements}

This work was supported by the NIHR Leicester Biomedical Research Centre, CAPEs, CNPq (n. 305616/2016-1 and 449467/2014-7) and FAPESP (n. 2017/00319-8). The authors would like to thank Prof. Eduardo T. Costa, Prof. Takashi Yoneyama and Dr. Fernanda Q. Rossi for their valuable suggestions.

\section{References}

[1] Haissaguerre M, Jais P, Shah DC, Takahashi A, Hocini M, Quiniou G, Garrigue S, Le Mouroux A, Le Metayer P, Clementy J. Spontaneous initiation of atrial fibrillation by ectopic beats originating in the pulmonary veins. N Engl J Med 1998;339(10):659-66.

[2] Nademanee K, McKenzie J, Kosar E, Schwab M, Sunsaneewitayakul B, Vasavakul T, Khunnawat C, Ngarmukos T. A new approach for catheter ablation of atrial fibrillation: Mapping of the electrophysiologic substrate. J Am Coll Cardiol 2004;43(11):2044-2053.

[3] Narayan SM, Krummen DE, Shivkumar K, Clopton P, Rappel WJ, Miller JM. Treatment of atrial fibrillation by the ablation of localized sources: Confirm (conventional ablation for atrial fibrillation with or without focal impulse and rotor modulation) trial. J Am Coll Cardiol 2012; 60(7):628-36.

[4] Verma A, Jiang CY, Betts TR, Chen J, Deisenhofer I, Mantovan R, Macle L, Morillo CA, Haverkamp W, Weerasooriya R, Albenque JP, Nardi S, Menardi E, Novak P, Sanders P, Investigators SAI. Approaches to catheter ablation for persistent atrial fibrillation. N Engl J Med 2015;372(19):181222.
[5] Steinberg JS, Shah Y, Bhatt A, Sichrovsky T, Arshad A, Hansinger E, Musat D. Focal impulse and rotor modulation: Acute procedural observations and extended clinical follow-up. Heart Rhythm 2017;14(2):192-197.

[6] Salinet JL, Tuan JH, Sandilands AJ, Stafford PJ, Schlindwein FS, André Ng G. Distinctive patterns of dominant frequency trajectory behavior in drug-refractory persistent atrial fibrillation. J Cardiovasc Electrophysiol 2013;25(4):371-379.

[7] Almeida TP, Chu GS, Salinet JL, Vanheusden FJ, Li X, Tuan JH, Stafford PJ, Ng GA, Schlindwein FS. Minimizing discordances in automated classification of fractionated electrograms in human persistent atrial fibrillation. Med Biol Eng Comput 2016;54(11):1695-1706.

[8] Almeida TP, Chu GS, Bell MJ, Li X, Salinet JL, Dastagir N, Tuan JH, Stafford PJ, André Ng G, Schlindwein FS. The temporal behavior and consistency of bipolar atrial electrograms in human persistent atrial fibrillation. Med Biol Eng Comput Jul 2017;

[9] Salinet J, Schlindwein FS, Stafford P, Almeida TP, Li X, Vanheusden FJ, Guillem MS, Ng GA. Propagation of meandering rotors surrounded by areas of high dominant frequency in persistent atrial fibrillation. Heart Rhythm 2017;14(9):1269-1278.

[10] Marwan N, Carmen Romano M, Thiel M, Kurths J. Recurrence plots for the analysis of complex systems. Physics Reports 2007;438(5):237-329.

[11] Webber CL, Zbilut JP. Dynamical assessment of physiological systems and states using recurrence plot strategies. Journal of Applied Physiology 1994;76(2):965-973.

[12] Uribe LFS, Fazanaro FI, Castellano G, Suyama R, Attux R, Cardozo E, Soriano DC. A Recurrence-Based Approach for Feature Extraction in Brain-Computer Interface Systems. Cham: Springer International Publishing. ISBN 978-3319-09531-8, 2014; 95-107.

[13] Soriano DC, Suyama R, Attux R. Blind extraction of chaotic sources from mixtures with stochastic signals based on recurrence quantification analysis. Digital Signal Processing 2011;21(3):417 - 426.

[14] Tuan J, Jeilan M, Kundu S, Nicolson W, Chung I, Stafford PJ, Ng GA. Regional fractionation and dominant frequency in persistent atrial fibrillation: effects of left atrial ablation and evidence of spatial relationship. Europace 2011;13(11):1550-1556.

[15] Almeida TP, Chu GS, Li X, Dastagir N, Tuan JH, Stafford PJ, Schlindwein FS, Ng GA. Atrial electrogram fractionation distribution before and after pulmonary vein isolation in human persistent atrial fibrillation-a retrospective multivariate statistical analysis. Frontiers in Physiology 2017; 8(589).

Address for correspondence:

Tiago Paggi de Almeida

Instituto Tecnológico de Aeronáutica

São José dos Campos, Brazil

E-mail: tiagopaggi@gmail.com 